\title{
EL CARRETE COMO ESCENARIO. Una aproximación etnográfica a los códigos de la sexualidad ocasional en jóvenes urbanos
}

\author{
CHRISTIAN MATUS MADRID*
}

\begin{abstract}
RESUMEN
La experiencia de la sexualidad en los espacios de diversión propios de la cultura juvenil es un fenómeno que ha sido muy pocas veces considerado a la hora de construir políticas preventivas dirigidas al mundo juvenil en Chile. Es en los escenarios propios de las cultura juveniles, asociados a sus espacios de ocio, particularmente los vinculados al «carrete», donde los jóvenes urbanos ensayan y ponen en escena diferentes experiencias de acercamiento a la sexualidad articulando códigos particulares: de construcción de intimidad en grupo, de relación con el tiempo y el espacio, y de acercamiento y aproximación al otro en el espacio festivo. El presente artículo está basado en los principales resultados de investigación etnográfica levantados por el investigador en el contexto del «Estudio de caracterización de los factores de riesgo y vulnerabilidad frente al VIH/SIDA en jóvenes», desarrollado a nivel nacional y que consideró una muestra integrada por jóvenes de sectores medios-bajos de las ciudades de Arica, Valparaíso, Santiago y Concepción.
\end{abstract}

PAlabRas ClaVe: JuVENTUd, Guiones SEXUALES, ETNOGRAFÍA

* Investigador Asociado de la Universidad de Chile, Santiago, Chile. Facultad de Ciencias Sociales. Antropólogo Social titulado en la Universidad de Chile, Diplomado en Crítica Cultural en la Universidad ARCIS, y es investigador en la temática de culturas juveniles. Agradezco en la elaboración del presente texto la valiosa e irremplazable colaboración de la antropóloga Loreto Navarrete Carrasco.

E-Mail: christianpau04@yahoo.es. 


\title{
O CARRETEL COMO CENARIO. \\ Uma aproximação etnográfica aos códigos da sexualidade ocasional em jovenes urbanos
}

\section{RESUMO}

A experiencia da sexualidade nos espaços de diversão próprios da cultura juvenil é um fenômeno que tem sido muito poucas vezes considerado na hora de construir políticas preventivas dirigidas al mundo juvenil no Chile. São nos cenários próprios da cultura juvenil, associados a seus espaços de ócio, particularmente os vinculados al «carrete», onde os jovens e as jovens urbanos/as ensaiam e poem em cena diferentes experiências de aproximação à sexualidade articulando códigos particulares: de construção de intimidade no grupo, de relação com o tempo e o espaço, e de aproximação a outro num espaço festivo. O presente artigo está baseado nos principais resultados de pesquisa etnográfica levantados pelo pesquisador no contexto do «Estudo de caracterização dos fatores de risco e vulnerabilidade frente ao HIV/AIDS nos jovens», desenvolvido a nivel nacional e considerou uma muestra integrada por jovens de setores médios-baixos das cidades de Arica, Valparaíso, Santiago y Concepción.

PALAVRAS CHAVE: JUVENTUDE, GUIAS SEXUAIS, ETNOGRAFIA

LEISURE-TIME.

An ethnographic approximation of the codes of occasional sexuality in urbanized youth

\begin{abstract}
The experience of sexuality in entertainment in the youth culture is a phenomenon which has not been very well considered within time to build preventive politics directed to the youth-world in Chile. In their very same scenario of the youth culture, associated with their leisure-time, where the urbanized youth pracitice and develop different experiences of approach to the sexuality articulating particular codes: building intimacy groups, together with time and space, and the approach and aproximation between each other in a festive space. The current article is based on the main results of ethografic investigation by the investigator in the context of «The study of the characteristics of risk and vulnerability of Aids in youth», developed on a national level and considered as an example integrated by youths of middle to lower classes sectors of the cities of Arica, Valparaiso, Santiago and Concepcion.
\end{abstract}

KEY WORDS: Youth, SEXUAL HYPHENS, ETHOGRAFIC 


\section{INTRODUCCIÓN}

LA EXPERIENCIA DE LA sexualidad en los espacios de diversión propios de la cultura juvenil es un fenómeno que ha sido muy pocas veces considerado a la hora de construir políticas preventivas dirigidas al mundo juvenil en Chile. Es en los escenarios de sociabilidad y encuentro propios de las culturas juveniles, particularmente los vinculados al «carrete», donde los jóvenes urbanos «ensayan»y ponen en escena diferentes experiencias de acercamiento a la sexualidad, susceptibles de ser leídas y articuladas en códigos particulares: de construcción de intimidad en grupo, de relación con el tiempo y el espacio, y de acercamiento y aproximación al otro en el espacio festivo.

\section{ACERCA DE RIESGOS Y JUVENTUDES}

El riesgo en el mundo juvenil posee una dimensión social y cultural innegable que adquiere especificidad en el marco de sus espacios de sociabilidad, sus experiencias de vida significativa y los códigos específicos de cada (sub)cultura juvenil siendo uno de los principales escenarios donde se expresa éste, el de la diversión y el «tiempo libre».

Es desde esta perspectiva que para aproximarse a la comprensión del riesgo en los jóvenes, es necesario adoptar una perspectiva que considere que observe a éste como un discurso que construido social y culturalmente es agenciado y resignificado de forma particular por los propios sujetos. Se requiere entonces el realizar una aproximación constructivista a la noción de riesgo.

\section{a) Una aproximación a la construcción cultural del riesgo}

La mirada constructivista acerca del riesgo intenta realizar una articulación entre los contextos culturales y la subjetividad. Según ella, las actitudes y valores asociados al riesgo estarían profundamente inmersos en un sistema de creencias, valores e ideales que constituyen a su vez una cultura o subcultura, que enfatizará ciertos riesgos y minimizará otros. No obstante, la perspectiva tradicional sobre éste ha tendido a ignorar que la percepción misma de riesgo y las maneras en que los actores sociales responden al mismo son moldeadas o construidas socialmente. 
El enfoque construccionista sostiene, en lo sustantivo, que los riesgos no constituyen ni propiedades objetivas que dependen de cómo sea físicamente el mundo, ni propiedades subjetivas que dependen de cómo sean cognitivamente los individuos. Los riesgos son construcciones sociales que dependen de factores socioculturales vinculados a estructuras sociales dadas. Pero tampoco son construcciones gratuitas, aunque sí convencionales, pues son entidades funcionales dentro de tales estructuras: permiten la distribución de la culpa y la responsabilidad, son utilizados como reclamo para la movilización social, permite la adaptación de la conducta individual a pautas colectivas marcadas por la opinión pública, etc. (López y Luján, 2000).

Es así como desde esta perspectiva, el riesgo se conceptualiza en un modelo dialéctico en el que están presentes la objetivación - la construcción del riesgo objetivo, del hecho en sí, del potencial nocivo del evento - y la desobjetivación — la construcción del riesgo subjetivo, los cálculos personales y grupales respecto a él, la percepción por parte de los individuos de la probabilidad de enfrentar un determinado riesgo-.

A diferencia de las teorías cognitivas, esta mirada no contempla la aceptación o no de riesgos tecnológicos como resultado de una decisión individual subjetiva. El enfoque es contextualizado, se centra en el estudio de los factores que hacen que determinados puntos de vista respecto a él, resulten dominantes en grupos sociales dados, o bien que se produzcan polarizaciones y enfrentamientos respecto de la distribución del riesgo.

Al interior de esta corriente, y desde una perspectiva antropológica Douglas y Wildawsky (1992) refieren que la noción de riesgo no se basa en evidencia empírica ni en razones prácticas, sino que está construida culturalmente de modo que, en cada contexto social, se destaquen unos riesgos y se ignoren otros. Existe así una cultura del riesgo asociada a la posición social de los actores haciendo que los peligros sean siempre identificados como tales, comunicados y gestionados a través de un filtro cultural.

Desde esta perspectiva, no puede entonces hablarse de expertos en temas de aceptabilidad de riesgos, pues no existe un punto de vista privilegiado para identificar y valorar riesgos. En este sentido, Douglas critica la frecuente acepción de la discrepancia entre riesgo objetivo y subjetivo, es decir, probabilidad matemática v/s probabilidad psicológica, como una cuestión de debilidad cognitiva. Los individuos 
no realizan coherentemente las selecciones que maximizarán sus ganancias esperadas o minimizarán sus pérdidas esperadas de acuerdo a la teoría de la decisión. Los peligros no son datos absolutos para elaborar una selección racional de los riesgos: existe una selección y una construcción social de los riesgos a través de los cuales éstos son moralizados y politizados (Paicheler, 1996).

Para Douglas, toda evaluación probabilista de acontecimientos - elemento proveniente de las teorías cognitivas del riesgo- opera en un contexto de incertidumbre. Ésta es más o menos bien tolerada y manejada por los individuos. La incertidumbre también da lugar a diferentes traducciones. Las incertidumbres serían fácilmente retranscritas en términos de certeza. En ese sentido, la selectividad y las contradicciones toleradas, usualmente son fuertes signos de proteger ciertos valores y las formas institucionales que los acompañan. Si el riesgo no es objetivamente definido por las personas en general, no es porque no haya logrado alcanzar ciertos criterios específicos idealizados, sino porque es intrínsecamente un concepto cultural.

De lo anterior, se desprende que si hay unas prácticas legítimas y por lo tanto objeto de atención y prevención respecto a la sexualidad juvenil hay otras carentes de legitimidad, que exponen a los jóvenes a una mayor vulnerabilidad y desprotección. En este estudio y fase etnográfica se intentó un acercamiento a aquellas prácticas de sexualidad ocasional vinculadas a contextos festivos que forman parte del «mundo del carrete», prácticas que por las condiciones contextuales en que se desarrollan son asociadas frecuentemente al riesgo de adquirir el $\mathrm{VIH} / \mathrm{SIDA}$. Pero antes de entrar a exponer los principales resultados de esta etnografía de la «sexualidad en el carrete», daremos una vuelta a la noción de riesgo desde el contexto de estudios e investigaciones sobre el mundo juvenil y sus prácticas de diversión.

\section{b) El riesgo en los contextos propios del mundo juvenil}

Pero para entender cómo se experimenta la noción de riesgo en el mundo juvenil es necesario acercarse al ámbito de los contextos y escenarios donde se desarrolla la vida cotidiana de los jóvenes. Es así como un esfuerzo por comprender las conductas de riesgo de los jóvenes en el ámbito de la sexualidad rebasa el estudio psicológico individual requiriendo, necesariamente, la inclusión de las condiciones del contexto y la trama simbólica y social en que se nutren las prácticas juveniles socialmente denominadas como «riesgosas» (Méndez Diz, 2001). 
Siguiendo una perspectiva constructivista, los resultados de recientes investigaciones en jóvenes (Matus et al., 2001) nos plantean que su concepción de riesgo no es unívoca sino que está determinada por las circunstancias en que ellos establecen sus proyectos de vida y los contextos culturales en que enmarcan sus experiencias significativas de sociabilidad y encuentro juvenil.

Un primer elemento que aportará a la comprensión de la noción de riesgo es entender cómo aparece este asociado a un cotidiano que tiene relación con su propio tiempo, su denominado «tiempo libre»o disponible para sí. Siguiendo esta línea, recientes estudios realizados en el marco de proyectos de intervención de reducción de riesgos asociados al mundo de las drogas, plantean la centralidad que adquiere el riesgo en los nuevos estilos de vida juvenil que se articulan en torno a los espacios de ocio festivo.

Como señala Funes (2004), los adolescentes y jóvenes de fines del siglo XX y comienzos del nuevo siglo construyen nuevas maneras de relacionarse con el alcohol, acceden a la cocaína, se divierten con anfetaminas, y formalizan un cierto uso de la cannabis (marihuana), todo esto en un contexto que configura grandes cambios.

Algunas de las transformaciones que caracterizan este nuevo contexto son: i) La consolidación de la adolescencia como etapa universal. ii) La generalización y masificación de determinadas pautas de ocio juvenil. iii) La concentración en el fin de semana, el predominio de la noche sobre el día, su asociación a determinados lugares, etc. iv) La creación de diversas culturas juveniles o los cambios en las anteriores, relacionadas con la diversión: predominio de determinadas músicas, estilos y estéticas, valoración de determinados estados de ánimo, etc. v) La presión comercial hacia el consumo y la generación de grandes negocios con el ocio juvenil. vi) El rechazo de determinadas drogas y determinadas formas de drogarse (el caso de la heroína en Europa). vii) La atracción de drogas, sometidas a modas y campañas de marketing formal o informal, y la mayor facilidad para sintetizar en laboratorio viejas y nuevas sustancias como la denominada MDMA (éxtasis).

Desde la misma perspectiva, De Rementería (2004) señala en forma más amplia - y para el caso chileno - que los jóvenes asumen cada vez más un conjunto de riesgos que tienen relación con sus procesos de desarrollo y aprendizajes temprano en la vida («riesgos atribuibles»). Es así como entre los 12 a 18 años se produciría la mayor tasa de morbilidad y mortalidad por accidentes caseros, en la vía pública, en deportes, en el 
campo, de tránsito, etc.; cifra que decae en el tramo de 18 a 24 años, lo que según este autor definiría la «vulnerabilidad propia de los jóvenes».

Por otro lado, en su «tiempo libre» los jóvenes realizarían un conjunto de actividades donde eventualmente se exponen a riesgos diversos como el recorrer lugares de diversión, recreación, esparcimiento, el uso de alcohol y drogas, las relaciones sexuales y el empleo de la violencia para la resolución de conflictos. Es así como sus comunidades locales - población, barrio, vecindario, grupos de pares, la familia-, las instituciones educativas, sus lugares de trabajo y los espacios de recreación y diversión, constituirían lo que el autor denomina ámbitos de «riesgo relativo».

Otro aspecto relevante tiene que ver con la actitud en relación al riesgo que asume el mundo juvenil, destacándose una valoración positiva del riesgo si el joven se encuentra inmerso en un contexto en que permanentemente se vivencia el riesgo, y una negativa si se está fuera de esos contextos.

Los jóvenes que actualmente están asumiendo riesgos, tanto los que actualmente consumen drogas como los que realizan deportes de alto riesgo, se refieren a sus acciones con una actitud positiva, en momentos eufórica; no es el caso de los ex-consumidores de drogas que hablan con amargura de su etapa adictiva, valorando negativamente sus acciones de riesgo. Parecería que al estar inmersos en los riesgos les hace tener una visión diferente que cuando se han apartado de ellos (Méndez Diz, 2001:151).

Por otro lado, y en relación con la realidad específica de los jóvenes de sectores populares, recientes investigaciones a nivel latinoamericano (Margulis, 2003) señalan que sus prácticas sexuales se desarrollan en situaciones precarias que promueven un tipo de sexualidad de contacto y más rápida, y en condiciones que dificultan la adopción de medidas de precaución o cuidado y potencian el desarrollo de conductas de riesgo.

Por lo general, las relaciones sexuales se desarrollan en condiciones precarias pues los jóvenes no disponen de espacios de intimidad. Así, la casa de los padres o de los amigos durante la ausencia de los mayores o zonas descampadas cercanas a la villa constituyen los lugares donde más frecuentemente se realizan los encuentros sexuales. En estas condiciones es posible pensar que la sexualidad asume una forma rápida y directa que entorpece las posibilidades de goce de las jóvenes más que la 
de los varones. Asimismo, es claro que tales condiciones alimentan las llamadas conductas de riesgo, pues una sexualidad así ejercida es contraria a las pausas que necesariamente requieren las prácticas de autocuidado. Y hay que agregar que los jóvenes de estos sectores, a pesar de que son muy conscientes de la amenaza del SIDA, son por lo general reacios al uso del preservativo. En este contexto, las chicas deben tomar la iniciativa para que su compañero lo utilice, pero cuando lo hacen no siempre lo logran y no son pocos los casos en que no lo exigen, con lo cual con frecuencia quedan embarazadas a edades tempranas (Margulis, 2003:192).

En relación al ámbito particular del «carrete», una reciente investigación desarrollada en Chile (Matus et al., 2001), nos plantea que desde la perspectiva de los jóvenes los riesgos forman parte de la vivencia y exploración propias de su vida cotidiana, y es en ese sentido que el riesgo forma parte de sus escenas festivas. En efecto, la exposición a peligros no sería exclusiva de los espacios de carrete, sino por el contrario, correspondería a una característica propia de todas y cada una de las situaciones de la vida en las cuales, de algún modo, siempre existe la posibilidad de ser dañado o perjudicado.

Si bien en el carrete se reconocen algunos factores que pueden favorecer una mayor exposición al riesgo, el acento presentado por los jóvenes en torno a su propia vulnerabilidad focalizaría en la necesidad de que existan condiciones que posibiliten un adecuado manejo y gestión frente a situaciones potencialmente riesgosas.

\section{EL CARRETE COMO CONTEXTO DE INVESTIGACIÓN}

El «carrete» constituye una de las prácticas más representativas y extendidas en la juventud chilena de postdictadura, siendo un ámbito de sociabilidad transversal a los diferentes estilos y culturas juveniles, estando definido por ellos como una de sus prácticas y espacios cotidianos más valorados (Matus et al., 2001; Silva, 1999).

En tanto parte de la cultura juvenil, el «carrete» está asociado a la forma particular en que los jóvenes se relacionan en el plano de lo festivo poseyendo una cualidad específica: ser un espacio al que se concurre en búsqueda de sentido, refuerzo e identidad. Es así como los jóvenes constituyen identidades y espacios de reconocimiento a partir de compartir espacios de «carrete» como pueden ser el recital, el estadio y el participar de fiestas realizadas tanto en espacios locales y barriales, como en espacios masivos como discoteques, gimnasios y otros (Silva, 1999; Contre- 
ras, 1996). Es considerando esta perspectiva que Contreras lo define como «la fiesta ritual, el encuentro transversal entre personas que poseen biografías fuertemente disímiles, que se descubren a sí mismas y a los otros como sujetos» (Contreras, 1996:56).

Uno de los supuestos centrales de nuestra investigación etnográfica fue el considerar el carrete como un espacio cultural que atraviesa transversalmente la vivencia de ser joven en Chile y que se encuentra directamente relacionado con las exploraciones que hace el mundo juvenil en el plano de la sexualidad. ${ }^{1}$ Es asumiendo esta perspectiva, que diseñamos una estrategia etnográfica para aproximarnos al «mundo del carrete» como espacio asociado a la sexualidad, dando cuenta de sus variantes y códigos a partir de la observación y registro realizado en diferentes espacios públicos y privados de las ciudades de Arica, Valparaíso, Santiago y Concepción. Así, asumimos el «carrete» como parte del guión cultural que ofrece nuestra sociedad a los jóvenes, en términos de constituir un escenario cultural, uno de los más relevantes mas no el único, que prescribe cómo los jóvenes se aproximan a la sexualidad.

Retomando lo señalado desde la Sociología de la sexualidad por Gagnon y Simon, todas nuestras experiencias sexuales estarían construidas como guiones, en el sentido que derivan de aprendizaje social, que no resultan tanto de la inculcación de las normas, de las reglas y de prohibiciones, sino que de una impregnación de relatos implicando secuencias de eventos e interiorización de formas de funcionamiento de las instituciones. El ser humano llegaría no sólo a un «saberhacer», como en todos los aprendizajes técnicos, sino también a una capacidad de percibir estados del cuerpo y a reconocer situaciones, desarrollando un aprendizaje que concierne a todos los aspectos de la sexualidad, incluyendo los eventos fisiológicos ligados a la excitación, el placer y el orgasmo (Gagnon, 1980).

Siguiendo a Gagnon y Simon, los «guiones sexuales» adquieren especificidad según los planos donde operan. Los «guiones intrapsíquicos» utilizan elementos de orígenes muy diversos, elementos simbólicos fragmentarios, escenarios culturales más ampliamente compartidos, elementos de experiencia personal, y los organizan en esquemas cognitivos estructurados que toman la forma de secuencias narrativas, de proyectos, de fantasías sexuales. Ellos coordinan la vida mental y el comportamiento social, y operan el reconocimiento de situaciones

1 Así lo señalan las investigaciones realizadas en torno al carrete en sí mismo Matus et al., 2001; Contreras, 1996. 
sexuales. Los «guiones interpersonales», principalmente presentes en el estado práctico de los diversos tipos de interacción social, se componen de secuencias ritualizadas y bien conocidas de actos que intervienen en el encuentro y en la seducción que provocan excitación y que coordinan la realización práctica de las relaciones sexuales. Existe evidentemente una interfase entre los guiones interpersonales y los guiones intrapsíquicos, en la medida que los últimos se constituyen ampliamente a partir de la memorización o de la anticipación de secuencias interpersonales.

Es en este contexto que los guiones de orden cultural o «escenarios culturales» son prescripciones colectivas que dicen lo posible así como lo que no debe ser en materia sexual. Estas recomendaciones y prohibiciones culturales nunca tienen, sin embargo, la simplicidad aparente de las normas y reglas legales. Son normalmente incluidas en relatos que no tienen necesariamente la sexualidad por objeto, o en funcionamientos institucionales que no aíslan siempre el aspecto sexual. En cuanto a los escenarios culturales que tratan explícitamente lo sexual, éstos no especifican solamente los objetos apropiados, las metas y las cualidades deseables de las relaciones entre sí y el otro, sino precisan también los momentos y los lugares, las secuencias de gestos y de propósitos y sobre todo lo que el actor y su (o sus) pareja(s) supuestamente van a sentir (Gagnon y Simon, 1984:105).

Es a partir de lo anterior que consideraremos como supuesto de investigación que el carrete constituye un escenario o guión cultural que, si bien no predice la conducta que los jóvenes van a tener en relación a la sexualidad, ${ }^{2}$ sí constituye un mundo en sí mismo que opera como referente de socialización interno y propio de la cultura juvenil en relación a la sexualidad, siendo un hito en la experiencia de ser joven, que atraviesa transversalmente diferentes estilos e identidades juveniles urbanas.

2 Cabe señalar que los escenarios culturales no son completamente predictivos de la realización de las conductas efectivas. Incluso si ellos constituyen la materia prima simbólica de lo sexual no funcionan sino haciéndose objeto de una interpretación por parte de los actores sociales, de un lado al nivel intrapsíquico y del otro al nivel interpersonal, al momento de la negociación de conductas. La dimensión intrapsíquica es un lugar de reformulación por parte de los individuos y de improvisación subjetiva a partir de los contenidos culturales cuyo ritmo de transformación es a veces muy lento. El nivel interpersonal representa el contexto social de la interacción, dentro del cual actores que no comparten siempre el consenso que existe en apariencia en el nivel cultural, se ponen de acuerdo sobre sus conductas. 
a) Dimensiones y variables abordadas

desde la aproximación etnográfica

Una vez descritos nuestros supuestos de investigación acerca del carrete procederemos a presentar las diferentes dimensiones y variables significativas que involucró nuestro trabajo de campo.

En primer lugar, la dimensión de la temporalidad. Nos pareció relevante acercarnos a la temporalidad cotidiana en que los jóvenes desarrollaban sus prácticas asociadas a la sexualidad. En ese sentido, consideramos como un espacio relevante el tiempo libre vinculado a la diversión y recreación y al «carrete», distinguiendo al interior de este ámbito, la temporalidad del día, la temporalidad de la noche, la temporalidad cotidiana de la sociabilidad y el encuentro cotidiano del grupo de pares, de la temporalidad festiva del evento y de la diversión que es experimentada como búsqueda de experiencias extracotidianas, imprevistas y no planificadas.

En segundo lugar, la dimensión de la espacialidad. Con esto nos referimos a los espacios físicos que determinan y configuran las condiciones y formas en que los jóvenes ejercen y practican su sexualidad. Es así como distinguimos en cada ámbito local-regional seleccionado la existencia de una geografía de lugares, tanto públicos como privados, en que los jóvenes desarrollan y ejercen su sexualidad (casa, espacios de calle, playas, cerros, locales de diversión nocturna y diurna).

En tercer lugar, la dimensión de la sociabilidad. Otra dimensión relevante en nuestro estudio tiene que ver con la centralidad que adquieren en el mundo juvenil los contextos de sociabilidad en que se reúnen, agrupan y conocen los jóvenes, siendo relevante conocer los sentidos y significados que los jóvenes construyen en relación con los espacios de sociabilidad, conociendo cuáles son los mayormente vinculados al ejercicio de la sexualidad.

Retomamos para esto algunas de las proposiciones de Maffesoli (1990) en relación al neotribalismo que nos plantea la emergencia de nuevos patrones de sociabilidad. La transformación en las relaciones sociales que Maffesoli define como neotribalismo emergente haría que el sujeto juvenil diluyera su experiencia cotidiana en la pertenencia a diferentes microgrupos o tribus.

Por último, dos dimensiones que se encuentran imbricadas, género y clase social. En efecto, dos dimensiones relevantes de considerar en la investigación son los atributos de género y clase. Es así como nuestro acercamiento al tema retoma la imbricación de los conceptos de género 
y clase social, distinguiendo la existencia de un conjunto de valores y significados asociados a la sexualidad que construyen diferencias en torno a lo femenino y masculino al interior de la cultura popular, significaciones y sentidos que son muchas veces retomados por las culturas sexuales juveniles de los jóvenes que forman parte de la muestra.

Cuadro resumen

Espacios asociados a la sexualidad

desde perspectiva etnográfica

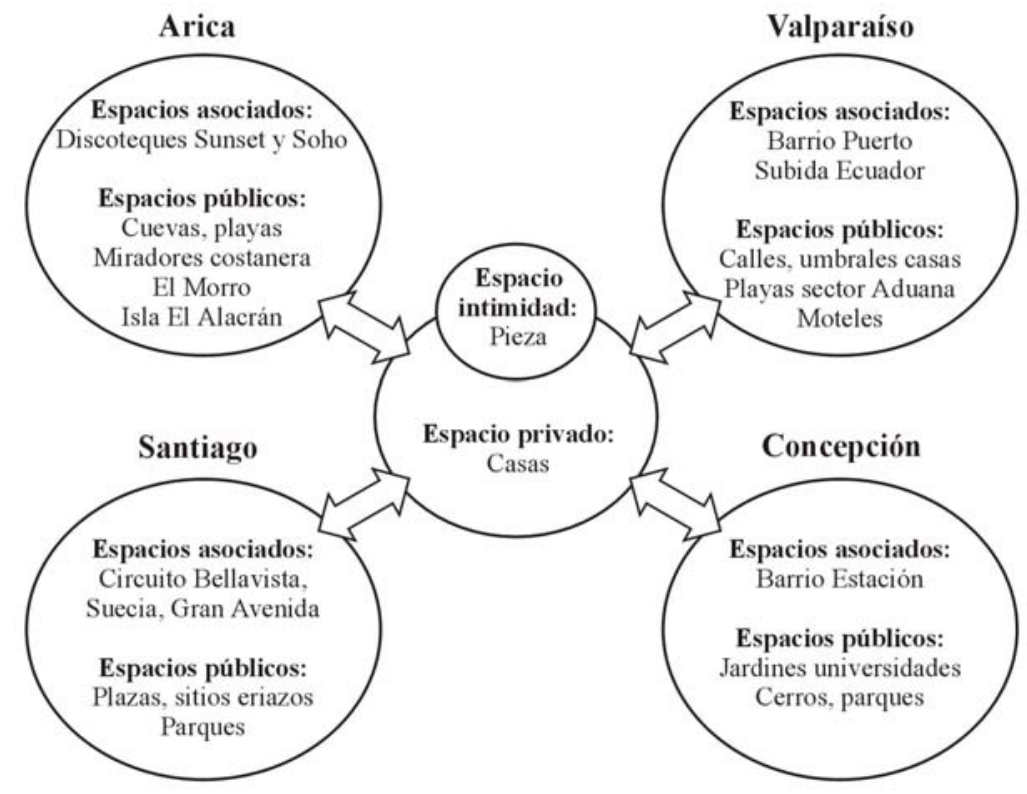

\section{ANÁLISIS INTEGRADO DE INFORMACIÓN ETNOGRÁFICA O LOS CÓDIGOS DE LA SEXUALIDAD ASOCIADA AL ESCENARIO DEL CARRETE}

El análisis de la información etnográfica nos permitió plantear la existencia de a lo menos cuatro códigos que caracterizan el vínculo carretesexualidad ocasional, códigos que en términos etnográficos son definidos como: de intimidad, de espacialidad, temporalidad y de seducción. 
a) Sociabilidad e intimidad. Del barrio a la ciudad o de la «intimidad de los próximos» a los encuentros intimos con «otros»

En primer lugar, se establece un vínculo entre intimidad y espacios de sociabilidad distinguiéndose diferentes ámbitos de encuentro juvenil en torno a los cuales se generan distintos tipos de vínculo y acercamiento a la intimidad sexual. Se distinguen tres espacios distintivos de vínculo entre sociabilidad e intimidad en el carrete que determinan cómo los jóvenes construyen su aproximación a la sexualidad ocasional.

Por un lado, el del carrete poblacional y barrial vinculado a la forma de ocupar el espacio que tienen los jóvenes de sectores populares, en el que la diversión se desarrolla en el barrio, en sus casas o en el espacio público de la calle, en las esquinas, en las plazas o los sitios eriazos del condominio o población. Este es el espacio de la proximidad donde la sociabilidad juvenil se lleva a la práctica en el grupo de semejantes, a nivel de las relaciones primarias, entre los vecinos y amigos, se trata de la sociabilidad entre los que comparten un modo de vida barrial. ${ }^{3}$ El ejemplo más concreto de esta experiencia que mezcla lo barrial con lo juvenil lo representa la aproximación etnográfica realizada en el sector de Plaza Waddington de Valparaíso, escenario particular en donde en un carrete se reúnen jóvenes hip-hoperos de diferentes sectores del Cerro Playa Ancha, actualizando la fuerte identidad del sector. El espacio festivo observado obedece claramente a una lógica barrial, lo que queda marcado en los cánticos que cada cierto tiempo irrumpen cuando se acaba la música que parecen imitar a una suerte de barra brava improvisada que en vez de alentar al equipo alienta al barrio: «Ohhh, yo soy playanchino, fumo yerba, tomo vino». En este caso confluye el estilo con la identidad barrial que es más fuerte que lo que los divide, su identificación con diferencias tendencias del hip-hop.

Es como vecinos y amigos que «vacilan la fiesta» porque todos son del barrio, y comparten un mismo modo de vida. Alejo, hiphopero, lo reafirma: «Playa Ancha es del barrio, aquí todos nos conocemos y escuchamos cumbia villera porque nos hace sentirnos del barrio» (Alejandro, etnografía Valparaíso).

3 Puede que existan diferentes estilos o modos de ser juvenil, pero antes que nada en el barrio prima la identidad de ser próximos y vecinos. 
En la población, ya sea de Cerro Playa Ancha, Cerro La Cruz, Barrio Norte o La Pintana, los jóvenes comparten cotidianamente en el marco de fiestas realizadas en sus casas, ya sea con el permiso de sus padres o sin él, lo que posibilitará que se desarrollen ocasionalmente «partusas», fiestas desbocadas, o «carretes duros» en los que el mundo juvenil aprovecha la ausencia del mundo adulto, existiendo siempre la posibilidad de tener relaciones sexuales en espacios como la pieza propia o del amigo, los baños u otros espacios de la casa.

No obstante, los que no tienen acceso a espacios íntimos donde tener sexo, desarrollarán una sexualidad asociada a los espacios públicos de la población (calle, sitios eriazos, plazas, etc.).

Un segundo ámbito de intimidad en el carrete que reporta el análisis etnográfico se encuentra constituido por espacios de sociabilidad intermedios caracterizados por agrupaciones y espacios de encuentro juveniles localizados más allá del ámbito específico de su residencia. Se trata de zonas y sectores de la ciudad (el carrete de la zona sur, el carrete de la zona norte en Santiago) que agrupan a un nivel más amplio de identidad que el directamente próximo de la población y el barrio, no obstante aún existe proximidad entre semejantes que pasan de ser directamente amigos a ser a lo menos «conocidos» porque comparten el mismo circuito por ser estudiantes de un mismo tipo de liceo o colegio o de la misma universidad o instituto o participantes de un mismo estilo juvenil. ${ }^{4}$

Esa relación de conocimiento mínimo generará la confianza para establecer nexos entre jóvenes que no se conocen directamente, pero comparten los códigos de un mismo modo de ser juvenil, otorgando un punto de partida para la seducción dentro de un marco de identidad compartida.

Un tercer espacio de carrete tiene relación con territorios más amplios que el barrio y la comuna, los que podemos definir como espacios de carrete urbano, o carrete a nivel de la ciudad. Constitu-

4 Estos circuitos de carrete tienen relación con los estilos juveniles siendo diferente el carrete desarrollado por los jóvenes que adhieren o tienen un gusto especial por el estilo «sound», que el carrete vinculado al circuito de las discoteques de música pop bailable en inglés. También se considera al interior de este espacio intermedio el ámbito del «carrete secundario» y «universitario» en donde adquiere mayor centralidad la identidad del joven como alumno de un colegio o una universidad, por sobre la identidad de origen vinculada al espacio del barrio. 
yen zonas de la ciudad en donde convergen jóvenes de una gran pluralidad de comunas y sectores. No son espacios directamente vinculados a las comunidades de origen (barrios, lugar de residencia) o grupos de referencia (circuitos y estilos juveniles) a los que pertenecen los jóvenes, sino que son territorios urbanos donde se concentra una amplia oferta de diversión juvenil en relación al carrete.

En particular, en el carrete juvenil asociado a centros de diversión nocturna se identifican dos espacios que convocan en forma masiva a los jóvenes y que tienen sus propios ritos y códigos. Son los espacios de las discoteques masivas y la apropiación y construcción de espacios de carrete de calle. Son los espacios más cercanos a la mayoría de los jóvenes, jóvenes tanto provenientes de sectores medios como de sectores con menos recursos para acceder a un espacio de carrete. Por otro lado, son también los espacios estigmatizados y asociados al estereotipo negativo del carrete por parte de los medios, producto del peso de los enfoques de seguridad ciudadana (Matus e Hidalgo, 2001).

En estos espacios urbanos se juega la posibilidad de vínculo en el carrete de jóvenes de distintos sectores de la ciudad que no se conocen previamente siendo posible y muy probable, encontrarse con «otros» y «otras» muy distintos al «nosotros» del barrio, del colegio, la universidad o el estilo local. Es así como el carrete de las discoteques implica la posibilidad de tejer nuevas redes sociales, la posibilidad de encuentros inesperados y de generar nuevas relaciones que impliquen la formación de parejas ocasionales y estables, de amistades, de nuevos vínculos. Al igual que en las otras dimensiones desarrolladas, y con mayor fuerza, se da la posibilidad de generar un tránsito de la socialidad a la intimidad, siendo los espacios de agenciamiento de esta sexualidad los moteles de la ciudad, el propio automóvil — si se tiene acceso - y los espacios públicos en menor medida.

Cabe señalar que un código distintivo del carrete urbano es la movilidad, la noción de recorrido por un circuito festivo, temporalidad de la diversión que también se transmite a la experiencia de la sexualidad que se hace más móvil y fluctuante, acelerada, que cuando se está en un espacio estable más próximo a la residencia.

\section{b) Espacialidad y sexualidad ocasional. Intimidad pública $y$ «lugares marcados» para la sexualidad}

Al hablar de espacialidad nos referimos a los espacios físicos que determinan y configuran las condiciones y formas en que los jóvenes 
ejercen y practican su sexualidad. Es así como distinguimos en cada espacio local-regional seleccionado la existencia de una geografía de espacios y lugares, tanto públicos como privados, en que los jóvenes desarrollan y ejercen su sexualidad (casa, espacios de calle, playas, cerros, locales y espacios de diversión nocturna y diurna).

En primer lugar, cabe hacer la distinción entre lugares de sociabilidad asociados a la sexualidad como los locales de diversión nocturna pubs y discoteques y los espacios de intimidad donde propiamente se realizan prácticas sexuales. Esto nos lleva a retomar lo señalado anteriormente en relación a la territorialidad, ya que si nos aproximamos a los espacios locales la sociabilidad e intimidad se encuentran casi siempre asociadas a un mismo espacio, la casa de un amigo, la propia, la plaza del barrio u otro lugar que se apropia como ámbito de intimidad. Esto es diferente en los espacios de carrete asociados al consumo cultural. Por lo general la mayoría de los jóvenes no tiene sexo directamente en el espacio físico de la discoteque (aunque puede darse el caso o puede desearse como fantasía), sino que transita de este escenario, de un ámbito de sociabilidad (casa de amigos, disco, pub) a uno de intimidad que puede ser nuevamente la casa, un motel o un automóvil si se cuenta con él, o en un caso extremo si no existe lugar ni acceso económico accede a tener sexo en un espacio público.

La experiencia ariqueña nos mostró cómo se desarrollaba la espacialidad asociada a contextos públicos. En efecto, encontramos una amplia y extensa geografía de espacios públicos que son apropiados por los jóvenes ariqueños como ámbitos de encuentro y diversión, de desarrollo y ejercicio de su sexualidad estableciendo los patrones de una intimidad en espacios públicos que conlleva una mayor dificultad de gestionar los riesgos que los de una sexualidad establecida en los marcos de los espacios tradicionalmente considerados como privados (la pieza, la casa, incluso el motel). Esto será especialmente crítico en espacios donde se construye una «intimidad pública» que queda ejemplificada en la apropiación que los jóvenes hacen de un espacio público como el de la Isla del Alacrán. ${ }^{5}$

5 La Isla del Alacrán constituye un archipiélago que se encuentra al frente del Morro de Arica en el sector sur de la ciudad de Arica; antes era una isla donde se instaló primero una cárcel y luego un centro pesquero, pero ahora se encuentra abandonada siendo apropiada por los jóvenes como un lugar de encuentro después del cierre de los locales de pub y discoteques y finalizados sus carretes. 
En el caso de Valparaíso, encontramos la coexistencia de ámbitos de diversión asociados a la cultura de los cerros donde se asientan las poblaciones y donde el carrete se realiza mayoritariamente en casas o en la calle, existiendo también espacios como Barrio Puerto, y en menor medida Subida Ecuador, donde se agrupan diferentes estilos de jóvenes alrededor de una oferta de distintos tipos de discoteque. También encontramos ya en el testimonio de los jóvenes que exploran con mayor asiduidad el ámbito del sexo ocasional la ocupación de espacios públicos propios de la geografía del puerto, que se encuentra marcada por la presencia de numerosos cerros en donde se puede construir una «intimidad pública».

Sí, por la arquitectura, de repente hay unos callejones cortitos ¿cachai?, sobre todo en los cerros, por ejemplo cerca de mi casa, yo vivo justo en una punta de diamante, entonces hay una calle pa'llá, una calle pa'cá, una escalera, esa escalera se divide en cuatro callejones, cinco callejones (Ricardo, etnografía Valparaíso).

También el contexto etnográfico de Valparaíso nos entregó antecedentes del vínculo que existe entre la elección del espacio y el entrenamiento del joven que busca sistemáticamente el encuentro ocasional, en ese sentido los lugares se acomodan a la necesidad de tener sexo en un contexto privado.

—En ese sentido por ejemplo ¿son ubicables los lugares para tirar?

- Yo cacho que hay gente que tiene lugares marcados, pero tú los buscai, o sea, te dai una vuelta por ejemplo cuando cachai que puede pasar algo, te dai una vuelta y cachai un lugar piola, unos árboles y hay harto recoveco, hay hartas casas ¿cachai? (Ricardo, etnografía Valparaíso).

Por su parte, en el centro de Santiago nos encontramos con una geografía de lugares y escenarios, que marcan distintas pautas de comportamiento en los jóvenes. Así es como comprendemos que el contexto espacial configura ciertas claves que se oponen a la de otros contextos. Paulina nos cuenta que reconociéndose una chica tímida, se siente libre de tomar la iniciativa en materia de conquista cuando se siente en su «propio espacio», el que se encuentra asociado al estilo juvenil gótico (Discoteque Blondie) del cual se siente parte ella, siendo muy distinto el comportamiento de Paulina si va a un escenario de carrete distinto como Barrio Suecia, espacio de carrete masivo, en donde no se siente a gusto ni comparte todos los códigos de aproximación y 
puesta en escena que «hay que tener» para seducir al otro. Un ejemplo distinto de esta relación y vínculo entre lugar/contexto de seducción lo encontramos con Gabriel, quien a pesar de compartir espacios de carrete con sus pares de «universidad privada», sale a buscar experiencias en un escenario distinto como es el de las discoteques masivas y populares del Barrio Bellavista siguiendo pautas de conquista y seducción diferentes a las que cotidianamente practica con su grupo más cercano. Como señala otro entrevistado, es el espacio, el contexto, la música, los que definirán la performance del género así como las pautas de relaciones con el otro.

Es interesante destacar como espacios de ejercicio de la sexualidad el motel y el automóvil. Tal como señalan Paulina y Marco, en Santiago existen espacios económicamente accesibles para los jóvenes, en el sector centro, en donde no se les piden ser mayores de 18 años, y se puede entrar con «copete» (alcohol) comprado en otra parte (ventajas importantes en comparación a la oferta de moteles para adultos). Cabe señalar que el espacio del motel aparece asociado en muchos testimonios etnográficos al ámbito de pareja más que al de la ocasionalidad, eligiendo muchos la emoción de lo ocasional en un sitio público o la seguridad que ofrece estar en una casa para tener sexo ocasional.

El automóvil, por otra parte, también será un espacio importante a la hora de ejercer la sexualidad, tanto por su importancia simbólica (significa y connota estatus en el caso de los hombres) como por la importancia práctica que adquiere, si no se tienen los recursos para pagar por un espacio para tener relaciones. El caso de la ciudad de Arica presenta una singularidad, ya que el automóvil, por su bajo costo a lo menos si es usado, aparece como un medio de transporte accesible para la mayoría de los jóvenes.

Por último encontramos que en Concepción se desarrolla una actividad mucho más ligada al ámbito de las casas en donde se realizan fiestas que pueden llevar o no asociada la posibilidad de tener sexo. Un factor determinante para los jóvenes es la movilidad ya que hay grandes diferencias entre los que tienen acceso a un vehículo en la región, jóvenes de clase media alta — que se divierten en discoteques ubicadas afuera de la ciudad - que tienen la posibilidad de acudir a un mayor número de espacios, que la mayoría de la población juvenil penquista que tiene que carretear en un circuito que remite a un solo lugar debido tanto a la falta de locomoción como a las condiciones climáticas. 
Aun así encontramos menciones a espacios públicos constituidos por los campus universitarios de la Universidad de Concepción y del Bío-Bío que proveen de espacios para tener sexo ocasional, lugares que se encuentran asociados a un uso tanto a escolares como universitarios.

No obstante, los mismos factores que determinan un repliegue de la socialidad del carrete hacia el mundo privado hacen que las pensiones universitarias y hogares de los jóvenes estudiantes que provienen de diferentes ciudades del sur de Chile sean también escenarios de encuentro sexual en el marco de carretes privados que reúnen a jóvenes que están recién constituyendo espacios de independencia en relación a sus familias, lo que hace que tengan mayor libertad para explorar en la sexualidad ocasional.

En síntesis, el aproximarnos a conocer los lugares y espacios donde los jóvenes tienen sexo en contextos ocasionales, la mayoría de las veces no protegidos, aparece como un aspecto relevante de conocer al momento de focalizar intervenciones preventivas (prevención primaria y secundaria VIH/SIDA) con públicos juveniles, siendo importante para promover una cultura de la prevención conocer las condiciones, el ambiente y los lugares y espacios concretos que los jóvenes eligen para tener sexo, dando cuenta de qué forma esta espacialidad considera o excluye la posibilidad de tener prácticas de sexo seguro y de llevar a la práctica la gestión del riesgo.

\section{c) Temporalidad festiva, de la no planeabilidad de lo extracotidiano a la relativización de la norma o el carrete como espacio más allá de lo discursivo}

Al aproximarnos a la temporalidad del tiempo libre vinculado a la diversión y recreación y al carrete, distinguimos y apreciamos la diferencia que existe entre la temporalidad del día y la temporalidad de la noche, la temporalidad diaria y cotidiana, asociada al día, pero también desarrollada las noches y fin de semana, donde se desarrolla la sociabilidad y el encuentro del grupo de pares, en oposición a la temporalidad festiva del evento y de la diversión que es experimentada como búsqueda de experiencias extracotidianas, imprevistas y no planificadas, que trascienden y dan sentido al orden cotidiano.

En nuestro recorrido, nos encontramos con espacios y escenarios en donde la temporalidad del carrete y de la fiesta es efectivamente experimentada como una búsqueda de experiencias extracotidianas, 
donde se pasa del «enganche» y de la conquista a la posibilidad de terminar la noche de fiesta teniendo relaciones sexuales no planificadas.

Los relatos de nuestros entrevistados así lo confirman, aunque las mujeres suelen negar que ellas busquen y tengan encuentros sexuales ocasionales. Creemos que en relación a ellas, hay un cierto discurso políticamente correcto que no da cuenta de cómo la experiencia de la ocasionalidad es agenciada por el mundo juvenil femenino, tendencia invisibilizadora que no puede ser superada fácilmente en una conversación de carácter informal y que requiere de estrategias de profundización más intensivas.

No obstante, cuando damos cuenta de la temporalidad del carrete estamos también ante una paradoja: abordamos un tiempo extracotidiano que cada vez se hace más cotidiano dentro del mundo juvenil, no obstante el carrete local o urbano opone sus códigos a los de la institucionalidad del colegio, la universidad y el hogar de distintas formas, instalando nuevas reglas a partir de la irrupción espontánea de la diversión en espacios vinculados al orden de lo productivo.

Un tipo de carrete que adquiere una dinámica distinta y que lejos de «rutinizarse» se caracteriza por su capacidad de disfrutar y gozar con intensidad carnavalesca el ámbito del «carrete», es el denominado «vacilón» que en las etnografías de Arica y Valparaíso aparece asociado a la diversión popular del mundo juvenil que vive en las poblaciones de los cerros. Los jóvenes al «vacilar» parecieran retomar el nexo con como el mundo popular vive lo festivo.

Por otro lado, si bien hemos definido al carrete como un espacio de exploración y búsqueda caracterizado por los atributos de lo no planificado que se encuentra fuera de los marcos de lo cotidiano, el espacio de la diversión festiva también cuenta con una forma particular de construir normatividad.

Más que normas/leyes, en el carrete la normatividad es relativa, asemejándose mucho más a las formas de construir normatividad que nos propone la lógica del juego. En efecto, el juego provee a los actores que «juegan» sus apuestas de ciertas reglas que son voluntariamente aceptadas y cuya transgresión no implica, en términos de sus efectos, la dramaticidad de la sanción.

En otras palabras, se pueden seguir las reglas del juego del carrete como se pueden abandonar no asumiendo la obligatoriedad del código normativo que plantea la ley. Incluso el mismo hecho de tener sexo queda dispuesto a un cierto azar, se puede participar, «tirar los dados», «apostar» pero no siempre se conjuga la «apuesta» con la 
ocasión: «cuando se puede, se puede, y cuando no se puede, no se puede» (etnografía Arica).

En ese sentido los jóvenes que buscan vivir una «ocasionalidad sistemática» serían «buenos jugadores», que leen bien los escenarios y saben cuándo realizar apuestas, considerando dentro del «software» o «programa» del juego el uso de condón.

En el carrete como juego, algunas reglas implícitas tienen relación con no «dar jugo», no embriagarse demasiado en términos que la seducción sea un acoso para el par femenino. El mismo consumo ritual de alcohol pareciera formar parte de la regla siempre que se provea su circulación al interior del grupo o de la pareja ocasional que emerge en el contexto del carrete.

No obstante la explicitación de los pasos a seguir para generar un acercamiento pareciera depender de los códigos del estilo y el escenario escogido para carretear. Otra regla sería no abordar («agujear», «mirar la mina») la pareja o posible pareja de otro en el contexto mismo de la escena del carrete, ya que una acción equívoca de este tipo usualmente genera la inmediata instalación de la violencia en el escenario de la diversión, siendo resuelta no en términos verbales, sino de agresión física.

Un punto importante para la comprensión de la lógica lúdica y las «reglas del carrete» en relación a la sexualidad, es entender cómo sus códigos subordinan lo discursivo al ámbito de lo simbólico. Dicho de otra forma, en un carrete las conversaciones e intercambios discursivos entre hombres y mujeres de distinto y mismo sexo, no obedecen a un fin lineal explicitado en el «texto» de la conversación, sino que más bien operan a nivel del «subtexto» o guión de aproximación, encuentro o «enganche», que los jóvenes representan/actúan de diferentes formas, pero con ciertas recurrencias en cada caso.

Por otro lado, los códigos festivos relativizan e invitan a transgredir la norma. Es así como una buena noche de carrete puede llevar a jóvenes que en su cotidiano adhieren a una normativa institucional a transgredirla. Una norma personal como podría ser la de «cuidarse», normatividad de la prevención que «obliga a usar condón siempre», puede ser «olvidada» o relegada, dejando al sujeto juvenil la interpretación de lo obligatorio en clave de juego y azar, lo mismo ocurrirá con otras normas/relatos culturales como la fidelidad a la pareja estable. 
d) De la «performance» al «enganche».

Los múltiples guiones interpersonales de la seducción

Otra dimensión significativa en nuestro estudio tiene que ver con la centralidad que adquieren en el mundo juvenil los contextos de sociabilidad en que se reúnen, agrupan y conocen los jóvenes, siendo relevante conocer los sentidos y significados que los jóvenes construyen en relación con los espacios de sociabilidad, conociendo cuáles son los mayormente vinculados al ejercicio de la sexualidad.

En relación con la dimensión de sociabilidad en el contexto del carrete, constatamos la existencia de diferentes microgrupos que se reúnen en torno a un estilo, una estética, con el propósito de generar y compartir sensaciones fuertes, así como proveerse de sentido. Tal es el caso de los jóvenes autodenominados «alternativos», góticos, hiphoperos, sounds, etc. En estos microgrupos, el ejercicio de la sexualidad será distinto y podrá adquirir ciertas particularidades de acuerdo al estilo o cultura juvenil. Por ejemplo, la diferencia entre los besos y encuentros sexuales fugaces en la oscuridad del ambiente alternativo se diferencia del guión más tradicional de conquista y seducción de las discoteques de Bellavista.

En relación con los diferentes tipos de estilos, cabe hacer la diferencia entre aquellos que tienen como espacio de vínculos los territorios locales del barrio («microculturas») de los que obedecen a las lógicas de encuentro urbano en espacios de consumo cultural, como las discoteques y pubs de la ciudad. Los primeros proveerán de contextos de relacionamiento colectivo a los jóvenes, en donde la sexualidad que explora la ocasionalidad se desarrolla al interior de un grupo de conocidos, donde se construye una intimidad basada en la relación de confianza que da el ser parte del grupo. Los segundos, espacios de búsqueda y «caza», requieren de un joven entrenado en los códigos de sexualidad asociados a la discoteque. Se trataría de una exploración en la ocasionalidad más sistemática en donde se asiste a un espacio presumiendo la posibilidad de un encuentro ocasional que desemboque en una relación de sexualidad ocasional.

No obstante, no todos los estilos potencian los mismos códigos de actuación y seducción. Es así como encontramos estilos que se articulan con concepciones de la sexualidad y el género tradicionales, que potencian la actividad masculina y la pasividad femenina restringiendo el repertorio de la exploración a la «caza masculina» (hip-hop, sound, por ejemplo). Encontramos otros estilos que se abren a la posi- 
bilidad de explorar femenina o potencian la indiferenciación masculina o femenina como código de seducción o la práctica de relaciones bisexuales. Estos estilos conforman lo que los jóvenes denominan espacios «alternativos». Un ejemplo de esto lo constituyen espacios como El Pagano en Valparaíso y el Bal Le Duc en Santiago.

Finalmente, el observar el carrete comprendiendo los códigos de vínculo juvenil nos permite relativizar la estigmatización que hace de éste el mundo adulto en términos de su reducción a un escenario de desobjetivación radical. En ese sentido, el carrete no es un espacio sin sujeto, sino un espacio intersubjetivo, en el sujeto juvenil no se «borra» ni se disuelve en el espacio festivo, sino que explora voluntariamente nuevos vínculos poniendo entre paréntesis - en mayor o menor medida - una dimensión de la identidad propia (la cotidiana) para generar el vínculo con los otros más próximos (barrio, sector) o más lejanos (en la ciudad).

La forma de establecer vínculo en el carrete está normada y preestablecida por el escenario cuyos códigos son voluntariamente aceptados y asumidos por parte de los que participan en la escena festiva, como señala Giorgio: «carrete y sexualidad van de la mano, pero depende de dos personas... de la mujer y del hombre. Si hay quórum, si hay feeling va a pasar algo» (etnografía Valparaíso). No obstante, varían las formas de interpretar y actuar estos códigos por parte de los actores hombres y mujeres que contraponen y contrastan los guiones culturales ofrecidos por el carrete con sus propios guiones, ya sean individuales o interpersonales.

Es así como hay actores que asumen el guión preescrito generando la actuación y puesta en escena correcta, adecuada de los códigos, ejecutando «el plan» previsto por el guión cultural.

El típico plan, o sea, agarrarse a una mina y llevársela donde podai (etnografía Santiago).

El plan es estar acá afuera y después ir a buscar mina allá adentro y salir a guerrear a la isla con las minas... la dura directamente... sincero (etnografía Arica).

Los discursos de los «ocasionales planificados» contrastan con los testimonios de las mujeres que parecieran «jugársela» por guiones muchos más abiertos a lo contextual y la improvisación — la «onda»o «lo que salga»—, no existiendo un deber ser, un rol fijo o una obliga- 
ción de actuar y cumplir un determinado papel en el escenario del carrete.

Yo creo que el carrete tiene demás vínculos con la sexualidad, yo creo que se forman ondas... no se está la movía si después de bailar enganchai con alguien, podís demás estar con él después o no estarlo, es variable, yo creo que depende de cómo tú estás con predisposición al carrete... no sé puh, si querís acostarte con alguien está como la pasá, está dado ahora, yo creo que va en que lo que buscai del carrete, las parejas sexuales que tu querís tener, la volá en que tú estai (Carla, etnografía Valparaíso).

Es así como si bien el guión tradicional, culturalmente asociado a lo masculino, pero que puede ser releído en clave femenina, gay o «alternativa», señala que es posible y probable «enganchar» con una «desconocida» en una discoteque para tener sexo, no siempre esta práctica se concreta o lleva a la práctica, porque colisiona con un guión personal distinto.

Acá todos vienen, enganchan y después quieren follar, si a mí me han invitado a follar un montón de veces, pero a mí no me gusta porque me da asco, no puedo tener sexo con alguien que no conozco. Si de hecho, mi último pololo con el que duramos un año y medio, que era mi amor de la vida, te digo, tuvo que pasar un mes y medio desde que nos conocimos para poder tener relaciones. Es que a mí me gusta el sexo con cariño, cuando hay una preocupación por el otro, podrá sonar muy mujer o muy gay pero así soy yo (Chico Alternativo, Bal Le Duc, etnografía Santiago).

También existen otras posibilidades y búsquedas de diversión que no necesariamente reproducen la modalidad socialmente promovida de divertirse, abriendo un espacio autónomo de diversión propio de las mujeres jóvenes. En efecto, en el «carrete femenino» aparentemente se produciría un desplazamiento de la sexualidad como práctica a la sexualidad como tema de conversación, abriendo un espacio colectivo $\mathrm{y}$ de género donde se puede reflexionar colectivamente desde lo cotidiano sobre la sexualidad como experiencia.

Claro que carreteamos entre mujeres, hablamos de sexo, de la vida, del amor, eh igual de... no sé, de los minos que son pasados de rollos. Los minos porteños de repente son medios barsudos, pero es lo que hay... pero todos son diferentes, yo creo que nosotras somos las únicas que no 
agarramos nada en la noche, porque nosotras salimos a pasarlo bien (Chicas en Barrio Puerto, etnografía Valparaíso).

Estas reformulaciones de los guiones tradicionalmente asociados a los roles de género femenino y masculino aluden a la forma en que los diferentes niveles de guiones contribuyen a estructurar la sexualidad existiendo siempre la posibilidad de generar nuevos ajustes entre un guión y otro.

Por último, para nuestro acercamiento a los guiones de seducción nos fue necesario retomar la imbricación de los conceptos de género y clase social, distinguiendo la existencia de un conjunto de valores y significados asociados a la sexualidad que construyen diferencias en torno a lo femenino y masculino al interior de la cultura popular, significaciones y sentidos que son muchas veces retomados por las culturas sexuales juveniles de los jóvenes que forman parte de la muestra.

Un primer hallazgo tiene que ver con el peso que asumen las representaciones de género en el carrete y su vínculo con la sexualidad. En Arica encontramos una fuerte presencia de representaciones de género que influyen en las prácticas de autocuidado y prevención. El imaginario juvenil masculino ariqueño establece y reproduce distinciones y estigmatizaciones sobre las mujeres jóvenes asociadas a prácticas más liberales sobre la sexualidad ocasional; estas distinciones no generan una estructura de comunicación de alteridad con la joven como una legítima otra que también tiene deseos y responsabilidades en relación con su sexualidad y cuerpo, lo que obstruye el que se instale el tema de la prevención al interior de la pareja ocasional - por decirlo de otra forma, no hay noción de «pareja» en lo ocasional pudiendo haberla - porque la sexualidad ocasional desde el mundo masculino $-\mathrm{y}$ probablemente también desde parte del mundo femenino a partir de un efecto-espejo- se construye en el marco de estrategias individuales que no consideran la noción de goce, complicidad y protección del sí mismo junto a otro. Por otro lado, la «performance de género» de algunos jóvenes como Gabriel (Santiago) nos hace recordar una masculinidad hegemónica fundada en la matriz de la hacienda, en que el joven sale en busca de sexo no planificado en otro escenario social, ajeno a su clase, en donde tendrá ventajas comparativas a la hora de la conquista y de conseguir relaciones sexuales con alguna joven.

Otro elemento a destacar, concordante con lo anterior, son las diferencias que encontramos en un mismo espacio al conversar con hombres y mujeres sobre cómo se hacen cargo o no de sus experien- 
cias sexuales en el carrete encontrando muchas veces una «subdeclaración de la ocasionalidad» en el mundo juvenil femenino. Mientras los hombres siempre comparten sus experiencias de sexualidad ocasional - ya que éstas tienen que ver con el prestigio de su masculinidad-, las mujeres suelen hablar más de los «otros» que de «sí mismas», trasladando el tema de lo ocasional a las «otras», las «más chicas», generaciones más jóvenes de mujeres, las que — en su opinióndesarrollarían prácticas de sexualidad ocasional.

e) «Amigas, yuntas, carreteras, vacilonas». Nuevos guiones de la sexualidad juvenil femenina

No obstante, si hurgamos en los testimonios de las mujeres jóvenes que adhieren a espacios de identidad autodenominados como «alternativos» encontramos experiencias que nos hablan de la emergencia de nuevas formas más «plásticas» y «líquidas» de relacionarse con la sexualidad. Estos nuevos tópicos de la sexualidad juvenil aparecen presentes en la trayectoria de vida sexual que nos narra Ana, una «chica» vinculada a la «escena carretera alternativa» de la ciudad de Valparaíso.

La sexualidad vivida por Ana determina el vínculo de su yo a múltiples alter egos, parejas pasadas, presentes y futuras que marcan y tejen un sentimiento de existencia social y personal de ella en tanto sujeto. Ella «transita» por identidades y estilos juveniles en forma simultánea — desde ser «punk rock» a ser «punk hippie»—, estilos que se hacen líquidos, moldeables al modo de ser joven que ella se plantea ser, aprendiendo con el tiempo a diferenciar la estética de la actitud: se puede cambiar de estética y seguir manteniendo un modo de ser joven contestataria. «Punky hippie... Fácil puh, fácil esa transición, es cambiarte de ropa, así de fácil, cambiai tu estética súper fácilmente. Te puedes poner una chaqueta de cuero y al otro día una de lana, pero la forma de ser nunca ha cambiado, siempre ha sido así, es autoestimularme, estimular a los otros, vacilar, no cambiar su forma de ser»».

Dentro de sus redes de relacionamiento, un contexto grupal que influye en su comportamiento - además de su identificación con un estilo como el punk- es el fuerte lazo que establece en el escenario del carrete con grupos de pares femeninas. Será junto con estas «amigas, yuntas, carreteras, vacilonas» que vivirá aprendizajes que le permitirán delinear su identidad sexual y personal y acceder a nuevas redes de vínculos. Primero será su aprendizaje de la femineidad a través de la imitación y mixtura en grupo de los códigos tradicionales y alternativos, combinan- 
do la imagen cultural clásica de lo femenino con una actitud agresiva propia de las imágenes culturales de los noventa.

Aparte que éramos cinco mujeres, las cinco éramos arianas, de Aries, entonces éramos como muy fuertes, teníamos un logo, teníamos todo un rollo, éramos un grupo de mujeres y nos reíamos y vacilábamos juntas, nos juntábamos en el colegio Lourdes... Como piño, éramos: sex sim$b o l$, como ese típico Spice Girls... Sí pues, completamente eran modelos... Pa'mí no, ahora son apestosas, por lo menos ahora, en este tiempo comprábamos... me gustaba esa femineidad como atrayente, sí, atrayente, atraía al sexo masculino, eran fuertes, cantantes, locas, tenían el pelo... no sé, tonteras, tonteras.

Esta misma «liquidez» de identidades que se moldean a las necesidades y contextos es la que vamos a encontrar en relación con sus experiencias y representación de roles y guiones en torno a la sexualidad, pudiendo ser femenina activa, «cazadora» y femenina pasiva - e incluso masculina - dependiendo de la expectativa del otro y el tipo de relación que quiera construir.

Por un lado, si analizamos la apropiación que Ana hace del escenario del carrete como espacio de conquista femenina encontramos que su actitud se asemeja mucho a la de los entrevistados masculinos que se apropian de los códigos para seducir y conseguir tener sexo ocasional.

Sí. El carrete, si yo lo tomo como un lugar de caza, si voy a cazar es un buen lugar, puedo observar, bacilar, comprarle un copete al loco, yo le compro copetes a los locos, cuando quiero cazar le compro un copete, lo invito, bailo, seduzco. El ritual, código mío si quiero conquistar a alguien... bailar, tocar, seducir, acosar, besar, empujar, arrinconar, beber, beber, beber mucho, toquetear; cuando es un ambiente de baile, hablar al oído.

No obstante, ella pareciera mostrar que el «salir de caza» femenino es distinto al tradicionalmente masculino, es un momento dentro de una trayectoria biográfica, «en ese rato cuando salía de caza era ese el código», que se sitúa en relación a un referente mayor, puede ser en el momento de una «pausa», la sexualidad como divertimento, placer, gusto sin la expectativa de pareja, apropiada un poco como «pausa», «intermedio» para salir del «imaginario de pareja» después de haber tenido una larga relación, siendo también un espacio de apertura a nuevas apuestas y posibilidades de encontrar/conocer a «nuevos otros». 
En síntesis, su tránsito y autoidentificación simultánea con distintos estilos juveniles como el «punk», el «mundo alternativo», y el ser mujer joven «vacilona» y «carretera», instala nuevas preguntas de investigación acerca del vínculo entre estilo, sexualidad e identidad personal en los jóvenes. ${ }^{6}$ El análisis de su trayectoria de vida sexual coloca en el tapete la necesidad de comprender la búsqueda y ensayo de diferentes identidades sexuales que promueven estilos juveniles emergentes como parte de procesos de autorreflexividad (Giddens, 1995), de interrogación y autoconocimiento de sí mismos que las nuevas generaciones desarrollan y constituyen a partir de la «apropiación» que hacen de sus biografías y trayectorias vitales en torno a la sexualidad. ${ }^{7}$

SANTIAGO (CHILE), ABRIL 2005

RECIBIDO: ABRIL 2005

ACEPTADO: JUNIO 2005

6 En ese sentido como señala el antropólogo colombiano Serrano (2003) en el mundo juvenil: «Lo importante de los estilos no son los estilos por sí mismos, como lo entendería la lectura sólida de las identidades, sino el cambio de un estilo a otro - a la manera de la mirada líquida - . Tras dichos cambios podemos encontrar las crónicas de las identidades contemporáneas, identidades que se alteran al momento de narrarse a sí mismas y que se dan gracias a la pluralización de los mundos de la vida, que es una condición de las sociedades post-tradicionales siguiendo también con la lectura de Giddens (1997). La presencia de los estilos supone la posibilidad de elección entre una serie de ofertas presentes, de mundos al alcance que están limitados por las condiciones de existencia de los sujetos» (Serrano, 2003:82).

7 En palabras del propio Giddens, «La identidad del ego se hace muy problemática en la vida social moderna, especialmente, en la época reciente. Rasgos fundamentales de una sociedad de elevada reflexividad son el carácter abierto de la autoidentidad y la naturaleza reflexiva del cuerpo. Para las mujeres que luchan por lograr una liberación de los papeles asignados a cada sexo, la pregunta ¿quién soy yo? emerge a la superficie con intensidad particular. Lo mismo sucede a los homosexuales masculinos o femeninos, que cuestionan los estereotipos heterosexuales dominantes. La cuestión es la identidad sexual, pero no sólo ella. Lo que los psicólogos anglosajones llaman el yo es hoy para cada uno un proyecto reflexivo: una interrogación más o menos continua de pasado, presente y futuro» (Giddens, 1995:38). 


\section{REFERENCIAS BIBLIOGRÁFICAS}

CONTRERAs, Daniel (1996): «Sujeto juvenil y espacios rituales de identidad». Proposiciones $\mathrm{N}^{\circ} 21$. Santiago: Ediciones SUR.

De REMENTERÍA, IBÁN (2004): «Presentación general». En IBÁN De REMENTERÍA (compilador): La reducción de daños y la gestión del riesgo en Europa y el carrete en Chile. Santiago: Red Chilena de Reducción de Daños.

Douglas, M. (1996): La aceptabilidad del riesgo según las ciencias sociales. Barcelona: Paidós.

y A. Wildavsky (1992): Risk and Culture. An essay on the Selection of technical and enviromental danger. Berkeley: University of California Press.

FUNES, JAUME (2004): «Drogas y adolescencia: dos iniciaciones simultáneas». En IBÁN DE REMENTERÍA (compilador): La deducción de daños y la gestión del riesgo en Europa y el carrete en Chile. Santiago: Red Chilena de Reducción de Daños.

GAGnON, J. (1980): Sexualidad y conducta social. México: Editorial Pax-México.

— y W. Simon (1984): «Sexual Scripts». Society No22. S/C: S/E.

y - (1973): Sexual Conduct, The Social Sources of Human Sexuality. Chicago: Aldine.

GIDDENS, A. (1995): La transformación de la intimidad. Sexualidad, amor y erotismo en las sociedades modernas. Madrid: Cátedra.

LóPez, J. A. y J. L. LuJÁn (2000): Ciencia y politica del riesgo. Madrid: Alianza.

MAFFesoli, Michel (1990): El tiempo de las tribus. El declive del individualismo en las sociedades de masas. Barcelona: Icaria.

MARGUlis, MARIO et al. (2003): Juventud, cultura, sexualidad. Buenos Aires: Biblos.

MATUS MADRID, Christian (2002): "Carrete juvenil y tiempo de ocio, inventando espacios (para ejercer el derecho a ser jóvenes)». Revista Patrimonio Cultural No26. Santiago: Biblioteca Nacional.

_ et al. (2001): «Noche viva: dichas y dichos del carrete juvenil». Cuadernos de Reflexión $\mathrm{N}^{\circ}$ 2. Santiago: ACHNU.

— y JAVIER HIDALGO (2001): «Entre consumos, dichas y riesgos: una mirada antropológica a la experiencia juvenil del carrete nocturno en el Barrio Bellavista». Ponencia Cuarto Congreso Nacional de Antropología, noviembre, Santiago.

PAICHELER, G. (1996): «Visiones del riesgo de transmisión del SIDA: incertidumbres y percepción del contagio». Seminario «Aporte de las ciencias sociales al enfrentamiento del SIDA», Santiago, noviembre, 1996.

SERRANO, JOSÉ (2003): «Es mejor ser líquido y no rígido. ¿Es posible una subjetividad juvenil?». En Ana Vergara y Juan Bustos (compiladores): Esa oscura vida radiante. Santiago: Universidad Diego Portales.

Silva, Juan Claudio (1999): Noventas. De maratones, vértigo y sospecha. Viña del Mar: Ediciones CIDPA. 